\title{
Acceso al suelo ejidal periurbano: análisis desde el capital social
}

\section{Access to mexican periurban social land: a social capital analytical approach}

\author{
SANDra Pola-Villaseñor* \\ YAdira MÉNDEZ-LeMUS* \\ ANTONIO VieYRA*
}

\begin{abstract}
Recently, access to land for residential purposes in Mexican peri-urban areas is undertaken through property rights transactions. The urban-rural context of the periurban fringe holds different land tenure systems making the land access a process where a mixture of formal and informal institutions, urban and rural agents, norms and legal frameworks coexist. This paper proposes an analytical approach based on the social capital to explore property rights transaction.
\end{abstract}

Keywords: access to land, social capital, parcelas, periurban fringe.

\section{Resumen}

Recientemente, el modo más recurrente para acceder al suelo ejidal en las periferias urbanas mexicanas es mediante mecanismos de compra-venta, estos procesos están caracterizados por la multiplicidad de actores, normas y marcos jurídicos, así como por la regulación de las instituciones formales e informales. En este trabajo se aborda dicho proceso a través del concepto de capital social, el cual ofrece elementos que permiten el reconocimiento y la sistematización en un contexto de periferia urbana convergente y transicional. Con base en una revisión de la literatura se hace una propuesta analítica de la compra-venta de tierras parceladas desde el capital social, se reconocen sus etapas, dimensiones, relaciones y elementos principales.

Palabras clave: acceso al suelo, tierras parceladas, capital social, periferias urbanas mexicanas.

* Universidad Nacional Autónoma de México, correos-e: sandrapolav@yahoo.com,ymendez@ cigaq.unam.mx, avieyra@ciga.unam.mx 


\section{Introducción}

Actualmente, en las ciudades mexicanas se registran crecimientos sin precedentes en términos demográficos y de superficie; los pobladores se establecen de forma más recurrente en la periferia de las ciudades, provocando que los límites urbanos se hagan cada vez más extensos y difusos. Debido a esto, en los espacios periféricos se manifiestan cambios constantes en el uso del suelo, principalmente de rural a urbano y frecuentemente fuera de la regulación urbanística. Las periferias se han convertido en lugares polinucleares y complejos donde confluyen elementos de los sistemas urbanos y rurales, producto de la multiplicidad de actores y de los intereses que ahí se confrontan.

Uno de los procesos clave para comprender el inicio de tales trasformaciones espaciales es el del acceso al suelo rural en la periferia urbana, ya que el modo en que se accede a él da cuenta de la naturaleza de los actores que participan, las instituciones que intervienen para su control, el nuevo uso que se le da al suelo y, por lo tanto, la manera en que se inicia la estructuración de la ciudad.

En México, después de las reformas al artículo 27 constitucional de 1992, la manera más recurrente para acceder al suelo en la periferia urbana mexicana es a través de acciones de compra-venta de tierras ejidales (Duhau y Giglia, 2008; Eibenschutz y Benlliure, 2009; Salazar, 2014). Sin embargo, hablar del acceso al suelo ejidal en la periferia urbana mediante mecanismos de compra-venta no resulta fácil, ya que por una lado las periferias son lugares que se encuentran en constante transición y en ellos convergen instituciones, formales e informales, así como estructuras sociales y actores de los contextos rurales y urbanos (Canabal, 2005; Cruz, 2005).

Por otro lado, la literatura evidencia que durante el proceso de acceso al suelo ejidal, las acciones de compra-venta son facilitadas o no por los vínculos construidos entre los actores y no únicamente por el beneficio económico que resulta de las transacciones (Hiernaux y Lindón, 1998; Eibenschutz y Benlliure, 2009; Salazar, 2014). Si bien el acceso al suelo ejidal en la periferia urbana ha sido estudiado desde diversas perspectivas, son necesarias nuevas formas de análisis que consideren a dicho proceso como un constructo social, con el fin de esclarecer dentro de las transacciones de los derechos de propiedad los vínculos y los mecanismos sociales que facilitan la privatización y el cambio de uso de suelo de rural a urbano y que lo caracterizan como un proceso que transita entre lo formal y lo informal.

Para tal fin, este trabajo propone un marco analítico para abordar el asunto de acceso a las tierras parceladas en la periferia urbana desde la 
perspectiva del capital social, el cual sirve como marco explicativo del proceso que involucra las fases que se atraviesan, los actores que participan, así como los mecanismos que facilitan el proceso que va más allá del establecimiento del precio como mecanismo de coordinación.

Por lo tanto, durante el desarrollo del texto se muestra la definición de acceso al suelo en la periferia urbana así como sus componentes principales, particularmente la importancia de la tenencia de la tierra y de las instituciones formales e informales. Enseguida se expone el contexto de la periferia mexicana y los antecedentes jurídicos y sociales que enmarcaban el acceso al suelo. Se expone el contexto actual y se recurre a las teorías económicas y de sociología económica para resaltar las características útiles para el análisis del proceso. Posteriormente se desarrolla el concepto de capital social que funge como elemento metodológico y explicativo del acceso al suelo en la periferia. Finalmente, se propone un análisis de los elementos y las etapas del acceso al suelo de tierras parceladas incorporando los elementos centrales del capital social.

\section{El acceso al suelo en las periferias urbanas: de la tenencia de la tierra y los niveles de formalidad}

Para acceder al suelo es necesario que los derechos asignados para el uso del mismo sean reconocidos por convenciones culturales, religiosas y jurídicas de una comunidad. Estos derechos son determinados por medio de los sistemas de tenencia de la tierra, que son instituciones sociales formales e informales creadas y estructuradas con base en las normas, los valores y las estructuras de poder de un grupo determinado (Portes, 2006).

Los sistemas de tenencia dictan quién puede utilizar qué recursos, durante cuánto tiempo y en qué condiciones (Canabal, 2005; Le Roy, 2007). La importancia de reconocer las categorías de tenencia de la tierra radica en que ésta dicta la vía que se lleva a cabo para acceder al suelo, por lo tanto se tiene implicación directa con la factibilidad de transferencia y la variedad de actores y de instituciones sociales que intervienen durante el proceso de acceso al suelo (Berry, 1989; Janvry y Sadoulet, 2001). La tenencia de la tierra, en tanto que institución social, tiene relación directa con los factores sociales, económicos, jurídicos, políticos, culturales de cada grupo social, por lo tanto, es de esperarse una gran complejidad en los sistemas existentes en las ciudades del mundo, además, cada uno tiene un nivel de formalidad dependiendo del marco institucional con que se regule (Le Roy, 1991; Payne, 2004).

Las instituciones que regulan los sistemas de tenencia de la tierra van desde lo formal a lo informal. En lo que se refiere a las instituciones formales se trata de reglas, procedimientos que son creados, comunicados e 
impuestos a través de canales ampliamente aceptados como oficiales. Esto incluye instituciones estatales, cortes, legislaturas y leyes (Helmke y Levitsky, 2004). Sin embargo, las informales, son menos claras ya que las reglas usualmente no están escritas y son creadas, comunicadas e impuestas fuera de los canales oficiales de sanción (Helmke y Levitsky, 2004). Esta definición abriría toda una gama de comportamientos o estructuras que podrían ser catalogadoss como instituciones informales. Sin embargo, una de las claves para reconocerlas es que la violación a lo determinado por una institución informal genera una desaprobación social o una sanción externa enraizada en las expectativas compartidas de los otros (Helmke y Levitsky, 2004).

Las instituciones informales no son menos válidas que las instituciones formales. Incluso Abramo (2012) niega la postura de los economistas institucionales, quien sugiere una subordinación de las instituciones informales ante las formales. De hecho, él sostiene que en realidad existe un entramado de instituciones informales producto de las instituciones históricas que regulan y sancionan las acciones. Al respecto LomnitzAdler (1988) asegura que no son producto de actividades aleatorias ni caóticas, sino que están basadas en redes informales consolidadas que siguen principios de lealtad, confianza, costumbre, y que funcionan paralelamente a las instituciones formales.

En la periferia urbana, hablar del acceso al suelo y de los sistemas de tenencia se complejiza, pues ahí se originan territorios, grupos, prácticas sociales "híbridas" y, como resultado, sistemas de tenencia de la tierra "híbridos" (Canabal, 2005; Cruz, 2005). Cuando se habla de rasgos "híbridos" se considera que los actores se remiten a instituciones rurales y urbanas, pero también a instituciones formales e informales, incluso se considera que los actores son capaces de reajustar sus estructuras a partir de la interacción social y modificarlas. Por lo tanto, es importante abordar el acceso al suelo no únicamente como un proceso dicotómico o dual, sino específico de un lugar, ya que es posible considerar que en las periferias urbanas los actores son totalmente diversos y son producto de procesos interrelacionados y específicos que, a su vez, producen espacios no homogéneos sino fragmentados (Cruz, 2005).

Particularmente, en las periferias de las ciudades de países en desarrollo, como es el caso de México, cada una de las categorías de tenencia de la tierra representa un subsistema que sirve a diferentes poblaciones con sus propias características, lo que promueve la proliferación de rutas de acceso y refuerza la diversificación de los usos que se le dan al suelo (Berry; 1989; Payne, 2004). En efecto, la diversidad da facilidad de acceso a más grupos y es posible pensar que facilita el proceso de acceso al suelo. Sin embargo, esto no siempre es benéfico porque se acarrean conflictos 
por la asignación de usos de suelo y se agudizan los problemas de aprovechamiento de recursos (Janvry y Sadoulet, 2001)

\section{El acceso al suelo en la periferia urbana mexicana: la adopción del dominio pleno y la privatización de tierras}

Explicado brevemente, puede decirse que el caso de la tenencia de la tierra en México tiene una historia de transmisiones no mercantiles antes del establecimiento de la propiedad privada. Fue hasta la Constitución de 1917 que se instauraron tres tipos de propiedad: la pública, la privada y la social (en este tipo están incluidas la ejidal y comunal). Autores expertos en el tema, como Cruz (2005), Yúnez-Naude (2006), RoblesBerlanga (2008), Salazar (2009) han detallado a profundidad las características de cada una de estas modalidades de propiedad, en especial las tierras ejidales; Sin embargo, en este texto se resalta lo estipulado en el artículo 27, donde se dictaba que los núcleos agrarios no tenían disposición libre de sus tierras ni el sector privado podía realizar acciones de compra-venta de forma legal, ya que la ley las definió como inalienables, inembargables e imprescriptibles. Por lo tanto, cualquier acción a manos de terceros era considerada como inexistente ante la ley.

Por lo anterior, alrededor de los años cuarenta, el acceso al suelo en la periferia urbana se hizo sobre tierras de propiedad privada (Azuela, 1989), pero a partir de los ańos cincuenta se produjeron barrios construidos sobre terrenos en la periferia que fueron adquiridos por vías legales e ilegales como invasiones, ocupaciones directas y ventas ilegales (Schteingart, 1989; Portes y Roberts, 2005). En 1992 se modificó el artículo 27 constitucional y como resultado se derogó la Ley Federal de la Reforma Agraria donde se impedía la venta de las tierras sociales. En el mismo año se publicó la nueva Ley Agraria y nuevas dependencias fueron creadas al interior del sector agrario ${ }^{1}$

Estas modificaciones al artículo 27 constitucional sentaron las bases para la consolidación de los mercados libres de tierra, que en teoría seguirían procedimientos formales y legales de cambio de tenencia de la tierra de ejidal a privada. Se sentaron nuevas vías de incorporación de tierras ejidales al desarrollo urbano, fueron dos acciones voluntarias ${ }^{2}$ por parte de los ejidatarios: la adopción del dominio pleno de las tierras par-

${ }^{1}$ Dependencias como el Registro Agrario Nacional (RAN), la Procuraduría Agraria (PA) y el Tribunal Agrario (TA). Mediante el Programa de Certificación de Derechos Ejidales (Procede) se expidieron los certificados y títulos de propiedad de la tenencia la tierra ejidal y comunal del país.

${ }^{2}$ En lo que se refiere a los solares urbanos les fueron expedidos títulos de propiedad y se les concedió pleno dominio de sus predios. Este tipo de terrenos puede ser vendido como propiedad privada y han aportado tierras a la expansión urbana de la periferia, sin embargo en ese trabajo no se ahondará en el estudio de sus ventas. 
celadas y la aportación de tierras de uso común a sociedades mercantiles. La primera consiste en que, una vez que se cuenta con el certificado parcelario, el titular puede solicitar a la asamblea ejidal la autorización para obtener el dominio pleno de su tierra. Si la resolución es favorable, el titular puede gestionar, ante el Registro Agrario Nacional, la cancelación de la inscripción de la parcela como ejidal e inscribirla en el Registro Público de la Propiedad. Así, las tierras dejan de ser ejidales y quedan reguladas por el derecho civil. En lo que se refiere a tierras de uso común la legislación considera la posibilidad de que ejidatarios y comuneros formen sociedades mercantiles inmobiliarias que permitan trabajar sobre desarrollos comerciales, fraccionamientos habitacionales, desarrollos turísticos y parques industriales (Cruz, 2005).

Después de más de una década, en 2008, 46\% del territorio mexicano $\left(909,941 \mathrm{~km}^{2}\right)$ fue certificado mediante el Procede como tierra de tenencia de la tierra social. Entonces, $67 \%$ de la superficie certificada se registró como tierra de uso común, $30 \%$ como tierra parcelada y sólo $1.1 \%$ como área de asentamientos humanos ${ }^{3}$ (Salazar, 2009). Del total nacional de tierras certificadas, $8 \%$ está ubicado en las periferias de las ciudades, de las cuales $50 \%$ es de uso común, $41 \%$ tierras parceladas y $2 \%$ asentamientos humanos; hasta el mismo año, sólo 5\% del total metropolitano había adoptado el dominio pleno (Salazar, 2009).

En lo que se refiere a tierras de uso común, autores como González y Nelly (2004) y Salazar (2014) tienen registros de que los ejidatarios han establecido sociedades mercantiles para aportar las tierras de uso común para proyectos, sin embargo, es un mecanismo que no ha sido muy utilizado y no abunda la información de datos nacionales. Bajo este panorama, es posible apreciar que los mecanismos para coordinar la tenencia de la tierra con el mercado no fueron utilizados para transformar la tenencia de la tierra, por el contrario, se establecieron las condiciones informales para la generación de nuevas modalidades para acceder al suelo (Clichevsky, 2000; Le Roy, 2007).

Actualmente, la principal modalidad de acceso al suelo ejidal en la periferia urbana mexicana es mediante acciones de compra-venta y se caracteriza por múltiples rasgos de informalidad. Incluso se reconoce que el proceso no es puramente informal, sino que los agentes actúan en uno y en otro según los costos o beneficios que cada uno ofrece (Clichevsky, 2000; Jaramillo, 2008; Salazar, 2014). A continuación, se ha elegido analizar la privatización de tierras parceladas debido a la disponibilidad de información y a que la acotación servirá para limitar la complejidad

\footnotetext{
${ }^{3}$ Los porcentajes han sido redondeados por fines prácticos. Para más precisión consultar Salazar, 2009 .
} 
del proceso así como para distinguir los actores y el tránsito que hacen del sector formal al informal, así como los mecanismos de origen y sostenimiento del mismo.

\subsection{Componentes de acceso al suelo}

De manera muy simple, los componentes del proceso de acceso al suelo en la periferia mediante compra-venta son la tierra, los actores -como compradores, vendedores y otros que se incluyen durante el proceso, como los intermediarios y los asesores-y las instituciones, que regulan y controlan el proceso que puede ser formal e informal. El cuadro 1 resume los atributos más importantes a considerar de dichos componentes.

La tierra. La oferta de suelo puede variar en cuanto a la cantidad de superficie disponible, la tenencia de la tierra, la ubicación del inmueble, el uso asignado por el Estado, las propiedades físicas. En este trabajo es de interés estudiar tanto las tierras parceladas, que tienen un uso originalmente agrícola con determinaciones urbanísticas de suelo en breña que no debe ser urbanizado, como el que sí es apto para ser urbanizado (Eibenschutz y Benlliure, 2009).

Los actores. Los vendedores: los ejidatarios o poseedores de la tierra son un elemento esencial en la expansión urbana y el acceso al suelo (cuadro 1). Según Salazar (2012), en la actualidad, los ejidatarios desempeñan un papel primordial, ya que el nuevo contexto constitucional ha incrementado el control que ellos tienen sobre el suelo de la periferia urbana. Appendini y De Luca (2006) describen a los campesinos mexicanos, entre ellos los ejidatarios, como personas de bajo nivel educativo y de edad avanzada sin acceso a créditos formales, con asesoramiento y condiciones de mercado limitado, lo que incrementa la dependencia con la tierra. Los compradores que se exploran en este estudio son aquéllos que pretenden cambiar el uso de la tierra de agrícola a urbano. Según Morales-Schechinger (2012) el tipo de comprador estará determinado por el uso final que le da al rural periurbano. ${ }^{4}$ Aquí se han clasificado a los compradores como particulares, a su vez divididos en inversionistas y patrimonialistas (cuadro 1). También, se identifican los promotores inmobiliarios. Enseguida a las dependencias de gobierno que adquieren reservas territoriales y finalmente las asociaciones civiles que dotan de suelo a sus miembros como parte de las movilizaciones sociales o prestaciones laborales (Schteingart, 1989). Además de los compradores y vendedores se han identificado a los intermediarios, los notarios y los asesores legales que intervienen durante el proceso.

\footnotetext{
${ }^{4}$ Aquí se ha trasladado el análisis de los vendedores a los compradores.
} 


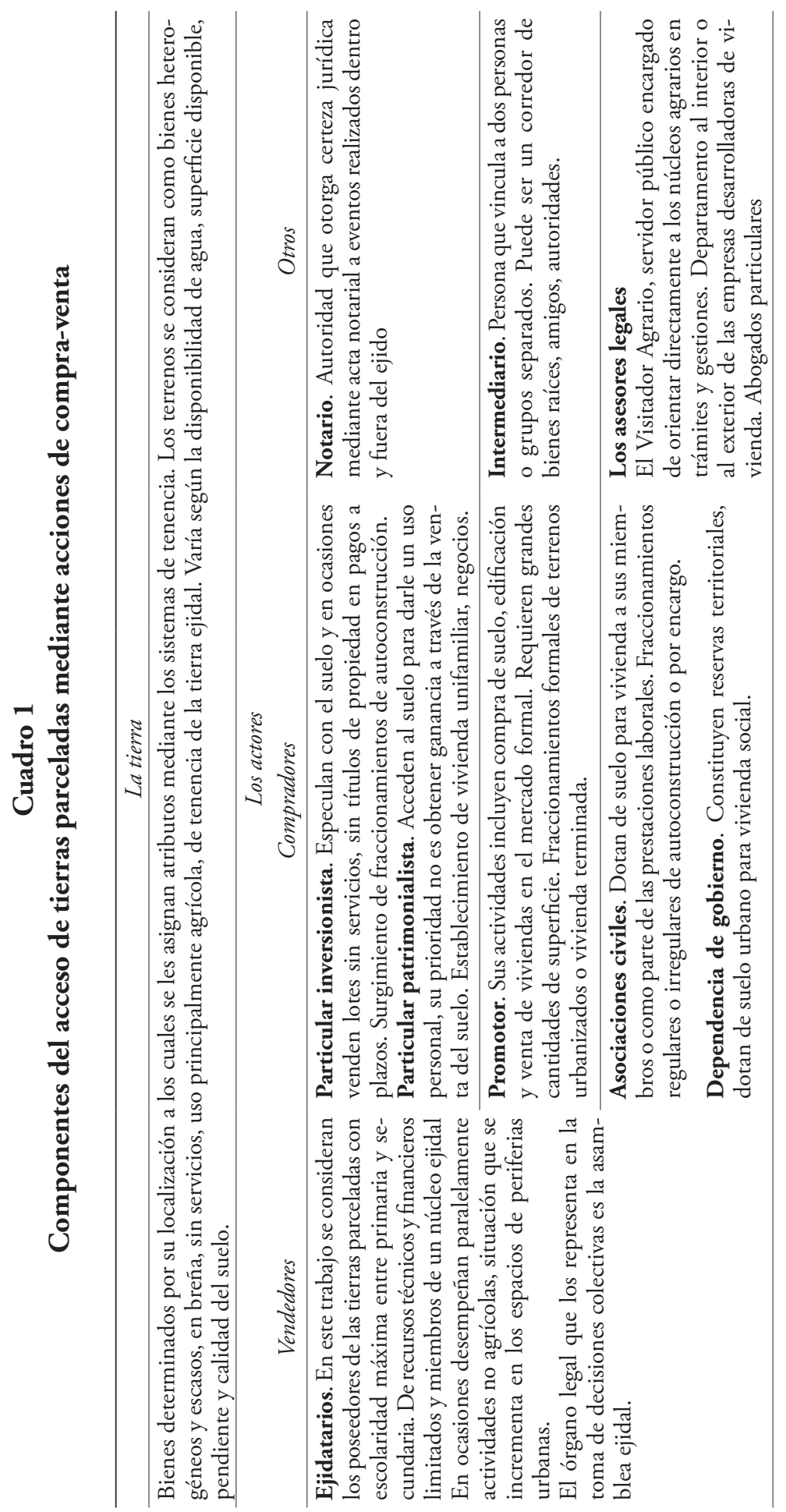




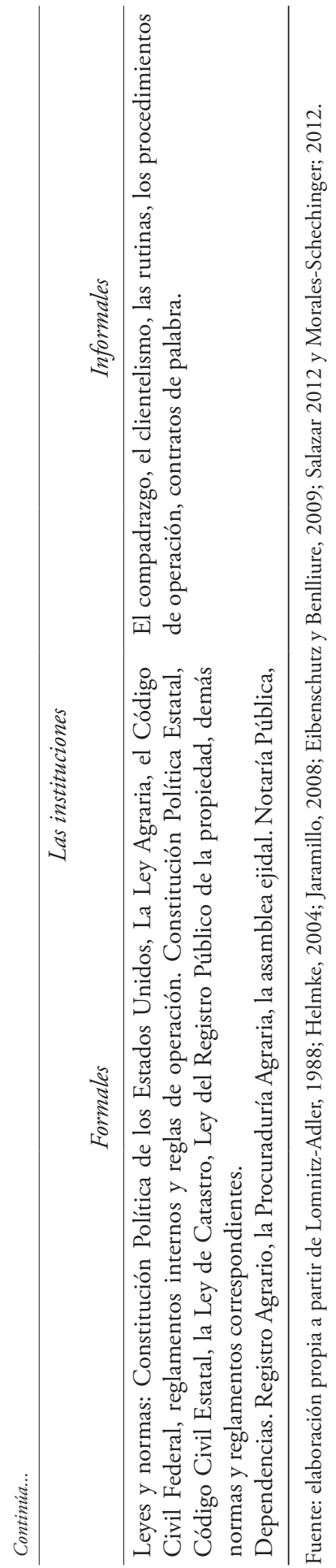


Las instituciones. Las instituciones formales que intervienen en las acciones de compra-venta de tierras parceladas son las leyes y normas correspondientes a la propiedad de la tierra y al derecho civil (cuadro 1). En cuanto a las instituciones informales en la literatura se reconocen el compadrazgo, el clientelismo, los contratos de palabra, los sistemas de pago.

\section{Acciones económicas empalmadas en contextos sociales: el capital social y sus elementos}

Para comenzar el análisis del acceso de tierras parceladas, desde la perspectiva del capital social, es necesario proponer una estructura analítica que tome en cuenta su empalme con un contexto social que considere a dicho proceso como un constructo social y no meramente como un intercambio mercantil. Esto ayudará a distinguir las etapas, los actores y las relaciones que se establecen entre ellos para logar acceder o no al suelo de la periferia urbana.

Desde una descripción proveniente de la economía clásica y neoclásica, el acceso al suelo mediante compra-venta es un intercambio mercantil $^{5}$ de los derechos de propiedad. Es importante precisar que las aproximaciones desde las teorías económicas han sido criticadas por el individualismo metodológico que supone y porque dejan de lado la historia, la especificidad de los lugares y la relación de los individuos con sus grupos o comunidades. Incluso, economistas como Coase (1960), Rutherford (2003), Hodgson (1988) y Lunn (2012) reconocieron la limitación que tienen las teorías económicas y, en efecto, se han complementado los modelos mediante la economía institucional, economía conductual, economía moral donde se han incluido elementos "extra económicos" como el papel de las reglas, las costumbres, las tradiciones, entre otros elementos que no dependen de los individuos involucrados (Rème, 2005). Sin embargo, Grandlgruber y Lara (2007), afirman que se obtiene una suerte de extensión refinada de la teoría neoclásica puesto que el aspecto normativo, así como la metodología individualista siguen rigiendo esta aproximación.

Para el objetivo de este trabajo, los fundamentos puros de estas teorías no son convenientes debido a las omisiones que se hacen en las dinámicas sociales. Sin embargo, las propuestas teóricas ofrecen elementos útiles para el estudio de las transacciones realizadas para acceder al suelo en la periferia. Por ejemplo, desde el marketing (Kotler y Armstrong, 2003) se consideran elementos como las dos partes que se encuentran, el bien

\footnotetext{
${ }^{5}$ Un intercambio en el que se establecen relaciones afectivamente neutras donde puede haber un encuentro breve y en el que posiblemente no continuarán las relaciones en el futuro. Se establecen acuerdos claros y definidos de tiempo y modo (Steiner, 2009).
} 
deseado que se intercambia y los mecanismos de coordinación que permiten el acuerdo en torno a las condiciones de entrega, de tiempo, de modo y de lugar. Asimismo, se hace referencia a las condiciones y las modalidades de la transacción, al contrato que garantiza el desempeño futuro de los convenios y al sistema de vigilancia de cumplimiento que puede ser la autoridad estatal o la moral (Williamson, 1981). En el marketing se considera que en caso de que no se logre un acuerdo, las partes tienen la capacidad de negociar para intentar encontrar un trato conveniente para ambos, de no ser así, las partes salen del proceso y no se culmina la transacción.

Por otra parte, los científicos sociales abordan las transacciones en cuestiones más concretas y dentro de condiciones contextuales determinadas a partir de la observación y de evidencias empíricas (Rème, 2005). La sociología económica, por ejemplo, ha intentado integrar los sistemas económicos a la organización social. Al respecto, Granovetter (1985) afirma que los actores, independientemente de su elección racional, son influenciados por costumbres, hábitos y normas que son seguidas mecánica y automáticamente. Años más tarde, él mismo (Granovetter, 2005) sostuvo que no hay una exclusividad en las acciones sino que en efecto existe un empalme (embeddedness) entre la acción económica y el contexto social.

Este trabajo concuerda con dicha afirmación en el sentido que las acciones no económicas tienen consecuencias en los resultados económicos y viceversa; y que, además, la estructura social en la que se lleva a cabo el intercambio determina las características de la transacción debido a que el estatus y las funciones de los agentes condicionan la asimetría en la transmisión de la información (Granovetter, 1985), la capacidad de negociación, los comportamientos deshonestos, así como las constricciones mentales de los actores fruto de su historia y sus influencias culturales (Williamson en North, 1995).

Al respecto, también Steiner (2009) sostiene que durante un intercambio mercantil hay casos donde la coordinación con el precio no es suficiente sino que se involucran intercambios no mercantiles para lograrlo. El autor explica que los vínculos personales y la movilización de recursos como la amistad y la confianza pueden intervenir y motivar la decisión de poner en venta un bien o incluso de modificar el precio para que se lleve a cabo la transacción. El mismo Granovetter (2005) menciona a la confianza como concepto unificador de las dimensiones económica y social. Por su parte, Aoki (2007), también reconoce que es necesario integrar la noción de confianza y de normas sociales a la acción económica, ya que elementos como la reputación, la honestidad y el intercambio de información aseguran el funcionamiento de los intercambios so- 
ciales y económicos. El mismo autor describe que en el "juego" de los intercambios sociales participan los actores canjeando símbolos sociales, acciones e información que, eventualmente, se convertirán en recompensas en una situación económica en la que se involucran los bienes materiales. Autores como Fligstein y Dauter (2006) ratifican esta postura diciendo que la confianza también involucra el poder y la dependencia, lo cual también reduce otras de las dificultades presentes durante las transacciones mercantiles que ya han sido anteriormente mencionadas.

Un concepto que cobró auge en los años noventa y que ayuda a explicar precisamente este empalme o embededness que según Granovetter existe entre las actividades económicas y no económicas, es el de capital social. Este concepto toma en cuenta la construcción y el mantenimiento de las relaciones sociales, así como el crédito social que se acumula durante las interacciones, el cual podría ser usado para obtener beneficios mercantiles o de cualquier otro tipo (Aoki, 2007; Portes, 2013). La ventaja del capital social como concepto unificador es que permite abordar tanto elementos económicos como no económicos en un mismo marco teórico y analítico, y a pesar de que el concepto ha sido ambiguo se han logrado avances en las explicaciones de los procesos mercantiles.

\subsection{Elementos centrales del capital social}

Autores como Bordieu (1986), Coleman (1988) y Putnam (1995) sentaron las bases del concepto. Sin embargo, en la actualidad no existe una definición única sino que su delimitación y definición sigue siendo ambigua (Woolcock, 2012). En este trabajo, el capital social es concebido como el cúmulo de relaciones con que un individuo o grupo de individuos cuenta, que están determinadas por normas formales e informales, valores, actitudes, niveles de confianza y principios de reciprocidad, mediante las cuales es posible acceder a ciertos beneficios individuales o comunes tangibles o intangibles (Bordieu, 1986; Krishna, 2007). De esta forma, se pretende integrar la dimensión económica y social en las acciones de los individuos en el caso particular del acceso al suelo ejidal en la periferia urbana.

El capital social comparte atributos con la definición de capital de los economistas (Grootaert y Van Bastelaer, 2001), por lo tanto es considerado como productivo (Coleman, 1988), acumulable y los beneficios que se desprenden de él dependen de su calidad, cantidad y diversidad (Grootaert y Van Bastelaer, 2001). Sin embargo, a diferencia de otros tipos de capital, el capital social no se aloja en los propios actores sino que es propio de la estructura de las relaciones entre ellos (Coleman, 1988). Es decir, que para que el capital social se active se requiere de al menos dos 
personas, y para lograr su mantenimiento son necesarias inversiones constantes, pues el desuso provoca un desgaste y detrimento de su calidad (Portes, 1998; Grootaert y Van Bastelaer, 2001).

Por lo anterior, Woolcock (1998) describe a la generación y al mantenimiento del capital social como un proceso iterativo que se inicia a nivel individual mediante mecanismos de expectativas y sentimientos de pertenencia que es sostenido por medio de contactos personales que desarrollan la confianza entre los vínculos, así como sentidos de reciprocidad y obligaciones con el otro, así como apego a las normas (Coleman, 1988). Para Mauss (1971), el origen y mantenimiento de estas relaciones sociales se lleva a cabo gracias al intercambio de bienes mediante la acción de dar, recibir y devolver un bien. A este bien se le adjudican cargas morales que eventualmente se convertirán en signos de reconocimiento a través de los cuales se crea un medio de comunicación que produce el conocimiento y reconocimiento mutuo entre los actores (Bordieu, 1980).

Por lo tanto, el intercambio es una suerte de inversión que refuerza el sentido de pertenencia con el otro (Bordieu, 1980), no de manera directa, sino como Durston (2002) lo explica: al iniciar un intercambio que es retribuido con un acto igualmente positivo, o aún más positivo, se pone en movimiento un círculo virtuoso con lo cual se refuerza el vínculo entre los actores. Eventualmente, tras una serie de repeticiones de interacciones positivas se producen y retroalimentan las relaciones de reciprocidad, confianza y cooperación (Ostrom, 2000; Durston, 2002; Arriagada, 2003). De esta manera, el individuo que inició el intercambio se coloca en una situación privilegiada donde, en teoría, ha obtenido la cooperación del otro o una suerte de crédito social, que podrá utilizar en otro momento y para el fin que crea necesario. Así, en una acción de repeticiones de interacciones positivas, se reproducen las relaciones de reciprocidad, cooperación y confianza, las cuales tendrán como resultado un refuerzo en el vínculo ya existente entre los actores (Arriagada, 2003). En caso contrario, el vínculo será desvanecido incluso eliminado.

Los bienes o activos movilizados entre los actores pueden ser materiales: riquezas, muebles e inmuebles, pero también inmateriales como información, gentilezas, favores, servicios (Mauss, 1971). La manera en que los activos se movilizan depende de las fuerzas sociales y morales (normas) cuyo motor son los mecanismos de reciprocidad y solidaridad (Narotsky, 2004). Cada comportamiento estará fuertemente definido por la cultura simbólica de las normas, de su carga emotiva, así como de creencias que surgen en torno a los roles y a las instituciones formales e informales que legitiman y producen la internalización de sus valores (Durston, 2002). Granovetter (1985) afirma que estos procesos se adquieren mecánica y 
automáticamente a manera de hábitos y costumbres derivados de una estructura creada por la historia y la continuidad de la comunidad.

\subsubsection{El capital social y las redes}

Cuando se dice que el capital social es propio de la estructura de los individuos es necesario aclarar que el capital social no son las redes ni la confianza ni las normas por sí mismas, pero es indispensable tener en cuenta que sin estos elementos no hay capital social (García-Valdecasas Medina, 2011). Es decir que las redes sociales fungen como la estructura potencial para reproducir y sostener el capital social y así crear nuevas redes. ${ }^{6}$

Las relaciones que se crean en las redes entre los individuos pueden ser formales -como la relación entre empresas, consejos de administración, sistemas de gobiernos regulados por instituciones formales y normas escritas-y también informales -como las de parentesco, afecto, interacción, vecindad, entre otras- (Requena-Santos, 1989). Según la teoría de redes, estos vínculos sirven como canales de comunicación por medio de los cuales se transmite información particular, incluyendo juicios de valor, soporte emocional, opiniones, mensajes, consejos políticos, pero también se intercambian instrumentos físicos o simbólicos, como regalos, servicios, compras, ventas, entre otros. (Requena-Santos, 1989).

Mediante el estudio de las redes sociales es posible ayudar a identificar el tipo de relaciones entre los individuos, así como los huecos estructurales originados por conflictos. Burt (1997) afirma que estos huecos estructurales en realidad son oportunidades para un tercero o "bróker" quien goza de la conexión de unas redes desvinculadas, por lo que puede intervenir para coordinar proyectos de ambas partes y controlar el flujo de éstas.

\subsubsection{Tipos de capital social}

A partir del estudio de la estructura de la red es posible identificar la jerarquía que guardan los individuos y, por lo tanto, el tipo de capital social que se genera. Por ejemplo la estructura del capital social considera al menos tres niveles de análisis: nivel micro, meso y macro (Grootaert y Van Bastelaert, 2001). Estos niveles determinan el tipo de relaciones que se generan: verticales y horizontales (Grootaert y Van Bastelaert, 2001), así como las transversales (Narayan, 1999). A su vez, el tipo de

\footnotetext{
${ }^{6}$ En este trabajo se entiende la estructura de los individuos como las redes sociales que han sido constituidas mediante actos sociales producto de la instauración y el mantenimiento de los vínculos durables y útiles (Bordieu, 1980).
} 
relaciones origina diversos tipos de capital social: como el capital de social de unión (bonding), el capital social de puente (bridging) y el capital social de conexión (linking). Durante las situaciones, es posible reconocer combinaciones del capital social de bonding, bridging and linking y que además serán cambiantes durante el tiempo debido a la búsqueda de una combinación óptima (Woolcock, 2001).

\section{El acceso al suelo en las periferias urbanas mexicanas desde la perspectiva del capital social}

Al partir de la definición teórica hecha en los apartados precedentes es posible retomar tres elementos principales para la estructuración y el análisis del proceso de acceso al suelo, desde la perspectiva del capital social: primero, que a partir de las teorías económicas de marketing y de transacción es conveniente dividir el proceso de compra-venta de tierras parceladas en las periferias urbanas en las etapas de entrada, encuentro, negociación y salida, así como resaltar los elementos principales de la transacción. Segundo, mediante la revisión bibliográfica se identificaron los actores principales que participan en el proceso de acceso al suelo en la periferia tales como los vendedores (ejidatarios), los compradores (las categorías expuestas anteriormente), los intermediarios y autoridades gubernamentales (notarios, visitador agrario, autoridades urbanas). Tercero, a partir del concepto de capital social y sus elementos se pretende resaltar las relaciones que se construyen entre ellos a diferentes niveles, así como los mecanismos sociales que habilitan las acciones de compraventa de este tipo de tierras (cuadro 2).

\subsection{El contexto social en el proceso de compraventa de tierras parceladas}

a) Etapa de entrada. El proceso inicia cuando compradores y vendedores están dispuestos a realizar acciones de compra-venta. Estudios han revelado (Hiernaux y Lindón, 1998; Eibenschutz y Benlliure, 2009; Abramo, 2012) que para encontrarse unos con los otros, cada uno de ellos recurre a amigos o familiares para obtener información de posibles clientes. Las relaciones están sostenidas por la confianza fundada en las normas, la costumbre, la reciprocidad y las interacciones en la comunidad. En este momento predominan las relaciones horizontales y es el capital social que se moviliza para acceder a los contactos entre las redes generalmente el de unión (bonding).

Debido a que en las redes de compradores y vendedores existen los "huecos estructurales" descritos por Burt (1997) aparece un actor que 


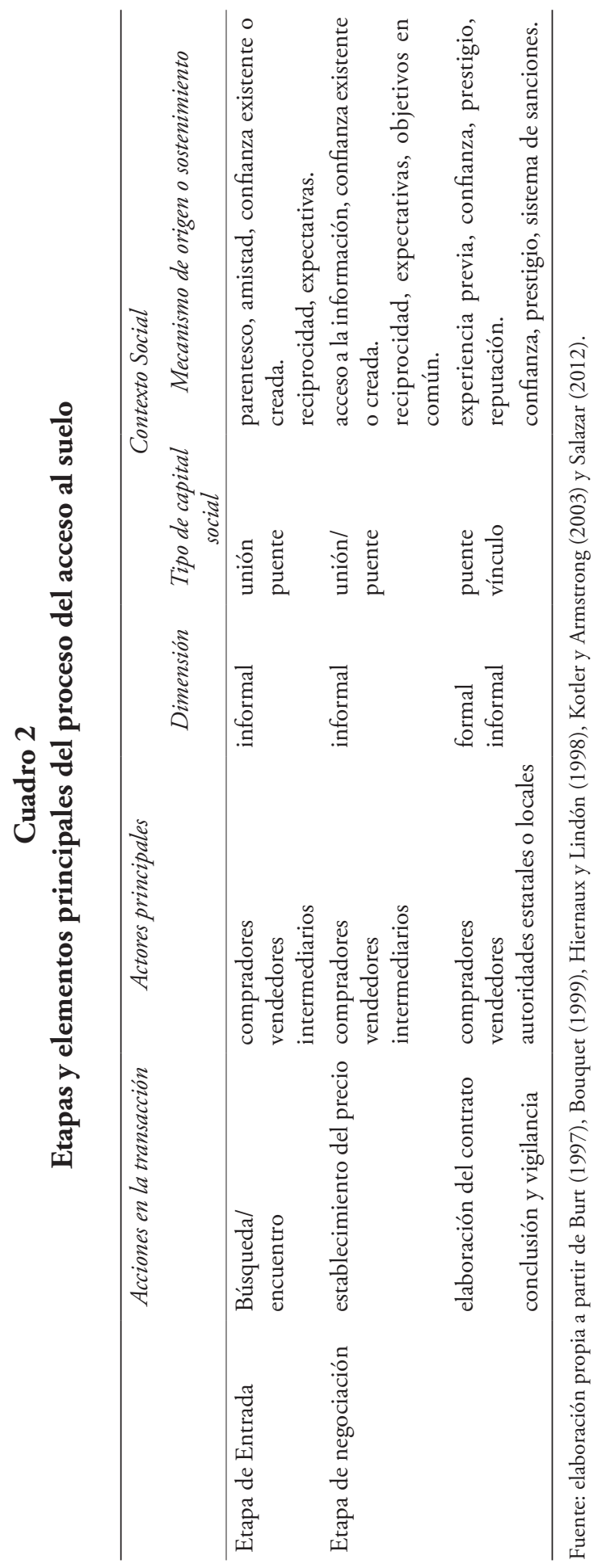




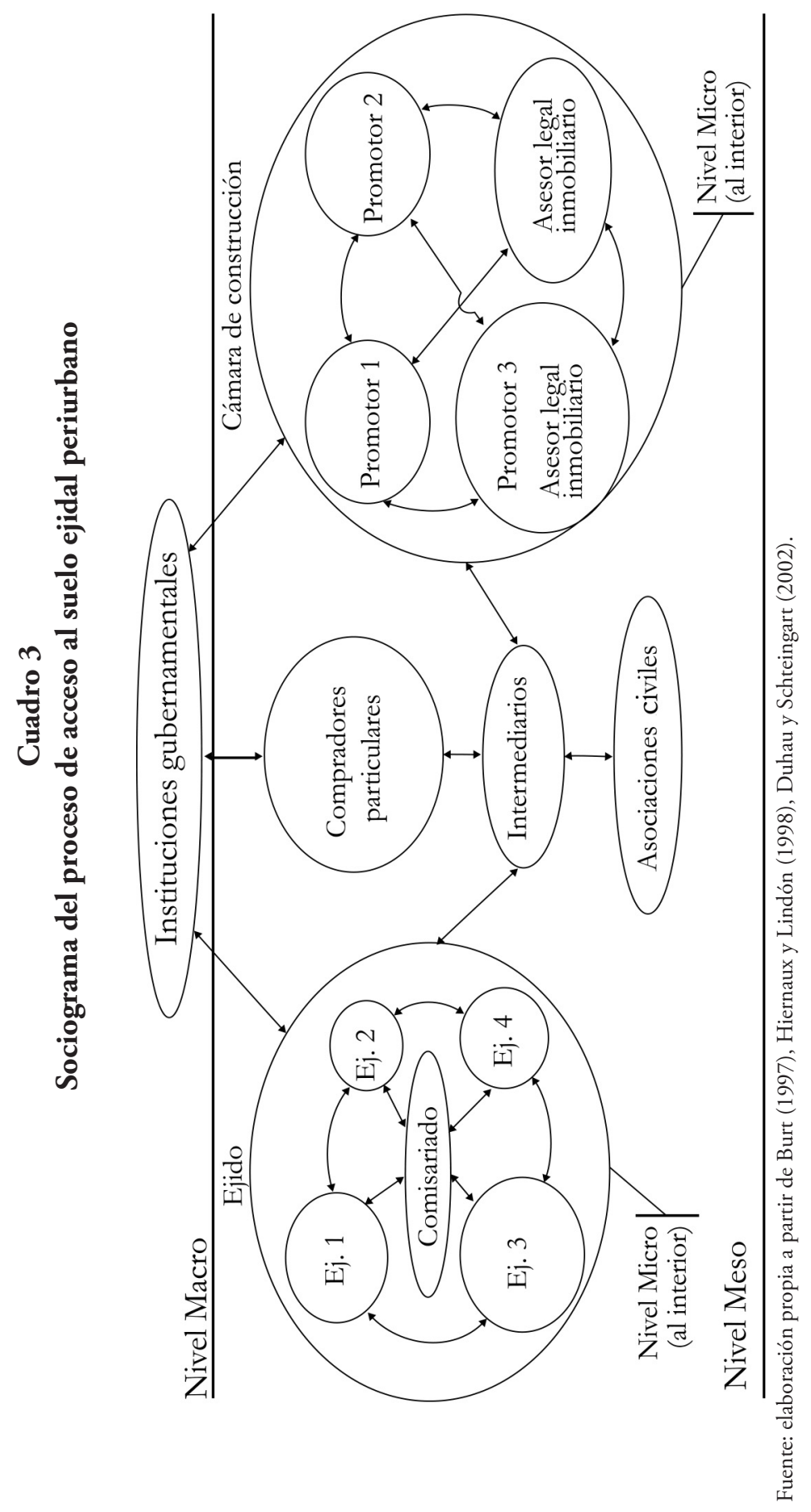


funge como intermediario (cuadros 2 y 3); regularmente es un amigo socio o conocido de las partes que cuenta con la confianza necesaria para entrelazar la relación entre ellos, estas personas están dedicadas a crear vínculos sociales como los corredores de bienes raíces, abogados o incluso representantes de las dependencias gubernamentales. Este tipo de actores se dedican a crear capital social de tipo unión (bonding) o de puente (bridging) entre las redes o actores desconectados, sea por la relación de amistad existente o por el contacto creado entre las partes.

Este capital social se construye a través de la confianza obtenida mediante encuentros frecuentes, promesas y, sobre todo, un elemento reconocido como un motor importante en la participación: el interés por realizar un objetivo en común. Durante esta etapa, el canal principal de intercambio es el informal, pues no abundan los registros donde intervengan las instituciones gubernamentales ni este tipo de relaciones se rige por las normas escritas.

Salazar (2012) afirma que la maniobra de las empresas inmobiliarias para contactar a los ejidatarios puede ser no propiamente a través de relaciones de amistad, pero en efecto, el contacto se basa principalmente en movilizar sus redes de relaciones para contactar a los comisariados ejidales. El establecimiento de este primer contacto es clave, pues esa persona, que sirvió como informante, constituirá un enlace para el establecimiento de relación de confianza que se ha definido como una "argamasa interpersonal" (Abramo, 2012).

Para los promotores inmobiliarios es conveniente contactar a los comisariados ejidales porque, a pesar de que los integrantes de las organizaciones ejidales tienen características económicas y socioculturales similares, éstos gozan de un cargo de poder que repercute hacia dentro de la organización a nivel micro y por lo tanto, influye los comportamientos dentro de un grupo, por ejemplo, la decisión de vender. La relación que se genera entre el ejido -en tanto que unidad organizacional-y los compradores -que en este caso son los promotores inmobiliarios- es de tipo puente. Las conexiones son de tipo transversal, dado que los actores tienen características disímiles y estatus diferentes pueden ser amigos distantes, socios (Woolcock, 2001).

Salazar (2012) describe que una vez hecho el contacto los compradores solicitan una reunión de la asamblea ejidal para exponer los servicios referentes a tres ámbitos: jurídico (asesorarlos sobre la normatividad federal, estatal, local), técnico (retomar la delimitación de los polígonos) y administrativo (gestionar ante las autoridades los trámites necesarios para la regularización de la tenencia y cambio de propietario). Además, hacen planteamientos acerca de las ganancias que los ejidatarios obtendrían al realizar una transacción con ellos. Esta declaración de bondad social es el 
llamado "velo ético" que oculta prácticas de dominación (GaribayOrozco y Balzaretti-Camacho, 2009) y es conveniente establecer una relación de confianza para esconder las intenciones utilitaristas y racionalistas de los promotores. En este momento se movilizan promesas y son empujados por los intereses comunes que generan compromisos y obligaciones recíprocas. Las relaciones que producen en este nivel son menos "fuertes" (por ejemplo con niveles de intimidad, confianza y frecuencia menores), pero los resultados económicos y sociales que se desprenden son en algunas ocasiones más productivos que los que se desprenden de relaciones llamadas "más fuertes" o de vínculo (Granovetter, 2005).

b) Etapa de negociación. Una vez establecida la primera relación de confianza, los compradores y vendedores comienzan la negociación para establecer el precio, el modo, el lugar y el tiempo de pago. En este momento es pertinente mencionar que tanto el comprador como el vendedor tienen capacidades de negociación diferentes debido a las características inherentes de cada uno, su rol en la estructura social, así como la calidad de sus vínculos que le otorgan acceso a información privilegiada (Callon, 2006).

En esta etapa influyen los tres tipos de capital social, por ejemplo, el capital social de unión (bonding) incide en la conexión entre amigos, colegas e instituciones formales y por lo tanto en el acceso y la calidad de la información que tiene influencia directa en la capacidad de cálculo del precio. En lo que se refiere al precio, Garibay y Balzaretti-Camacho (2009) afirman que los ejidatarios valúan la tierra en términos agrícolas, y con la noción de riqueza que sus recursos culturales y cotidianos les otorga.

Por otra parte, Eibenschutz y Benlliure (2009) argumentan que los compradores conocen las cotizaciones globales y el precio de la tierra se establece en función del valor de la vivienda terminada y del margen de ganancia deseado. Asimismo, los promotores inmobiliarios obtienen información local privilegiada de sus contactos en las instituciones formales. Al respecto Hiernaux y Lindón (1998) afirman que en ocasiones los promotores, especuladores e intermediarios están estrechamente ligados a la estructura clientelista del partido oficial incluso ocupando cargos administrativos locales. Estas relaciones siguen siendo verticales de tipo conexión cuando los intercambios se hacen en un ámbito informal que es menos evidente y más exclusivo.

Asimismo, estos actores están inscritos a una cámara o colegio donde las empresas constructoras comparten información y toman decisiones por su bien común. Los promotores inmobiliarios también tienen capital social de tipo unión, puente y enlace al interior, pues cuentan con departamentos altamente especializados y sus estructuras organizacionales facilitan la movilización vertical, horizontal y transversal de la información, 
así como la coordinación y colaboración formal e informal para la toma de decisiones. Por ejemplo, el departamento jurídico dedicado exclusivamente a las negociaciones y a la ayuda de gestión de trámites legales (Hiernaux y Lindón, 1998; Duhau y Schteingart, 2002; Cruz, 2005; Lomnitz-Adler y Sheinbaum, 2011).

El capital social de unión (bonding) que existe al interior del ejido también influye en la capacidad que tienen los ejidatarios para aceptar o no una oferta, ya que de la calidad de las relaciones depende de su capacidad de decidir en grupo. Garibay-Orozco y Balzaretti-Camacho (2009) exponen que las instituciones agrarias usualmente están regidas por la igualdad, el prestigio o la clase y se ven modificadas por prácticas de convencimiento y coacción provenientes del exterior, en este caso por los compradores. Duhau y Schteingart (2002) señalan que en ocasiones al interior del ejido se produce una diferenciación social significativa ya que no todos los miembros cuentan con la misma capacidad de decidir ni tampoco todos obtienen la misma información o los mismos beneficios. Esto, porque hay casos donde los comisariados ejidales y el comité de vigilancia disfrutan de un amplio margen de acción para dirigir y decidir sobre los asuntos del ejido.

Otro elemento que intensifica la asimetría entre las partes es la experiencia que los actores tengan en comprar o vender tierras, pues hay ejidatarios que nunca han tenido la experiencia de vender sus tierras, pero hay quienes echan a andar su capital social de unión y recurren a la búsqueda de información entre amigos o vecinos para hacerse de capital social de puente; incluso hay algunos que se han convertido, ellos mismos, en fraccionadores de las parcelas (Hiernaux y Lindón, 1998; Duhau y Schteingart, 2002). También existen algunos comisariados ejidales relacionados con actores en posiciones de poder favorables como abogados o funcionarios públicos (privados o el visitador agrario). Estas relaciones son de tipo vertical y se dan a nivel meso o macro y pueden ser de tipo formal o informal, por lo que los mecanismos de generación y sostenimiento son diferentes. Es pertinente resaltar que en todos los niveles existen combinaciones de los tipos de relaciones y por lo tanto del tipo de capital social que está presente (Woolcock, 2001).

c) Etapa de conclusión y vigilancia. Una vez aceptada la venta y el establecimiento del precio, los actores proceden a la determinación del lugar, el tiempo y la modalidad de la transacción. Según Bouquet (1999) los acuerdos alcanzados durante la compra-venta de tierras se remiten a diversos marcos de reglas. Por ejemplo, la Ley Agraria no permite la venta de los derechos parcelarios, a menos de que se haya optado el dominio pleno, así que en ocasiones, en lugar de un contrato escrito formal basta con un acuerdo a la palabra que legitima y vigila el proceso basado en 
relaciones de confianza mutua para que el ejidatario ceda su certificado parcelario a nombre del vendedor (Bouquet, 1999; Cruz y Chávez, 2012).

De no ser así, los vendedores hacen un acuerdo de compra-venta ex ante entre las partes, acuerdo regido por el derecho civil (Salazar, 2012); una vez cedido el derecho parcelario mediante el derecho al tanto, y con la elaboración del contrato de compra-venta, el comprador procede a hacer el pago antes de que la venta haya sido registrada por un notario ya que en ocasiones los ejidatarios reciben un pago diferido en cuotas y se registra hasta haber saldado la deuda (Salazar, 2012). En este momento, el comprador está en condiciones de iniciar los trámites del dominio pleno. Sin embargo, hay casos donde no se tramita el dominio pleno e, incluso, en esta fase se realizan ventas a terceros. Esto genera irregularidad en la tenencia de la tierra y para su incorporación el gobierno interviene mediante mecanismos de regularización (Salazar, 2014).

Ante la Ley Agraria esta clase de acciones no tienen validez, sin embargo, los documentos fungen como símbolos "formales" de vigilancia con el fin de continuar con la transacción. Al respecto, Azuela (1989) afirma que durante el momento de las negociaciones, en las prácticas de compra-venta los actores no buscan alejarse del orden estatal, sino al contrario, fundamentarse en reglamentos y leyes gubernamentales, al menos, para invocar su poder de legitimación y acceder a las instituciones formales en caso de algún conflicto, y con ello, tener la "certeza" de que el estado solucionará su caso.

Estas prácticas resultan legitimadas por las operaciones que todos los involucrados admiten como válidas mediante confianza y expectativas, aun si la Ley Agraria no avala este tipo de acciones (Duhau y Schteingart; 2002), y, en efecto, permiten la transacción de los derechos parcelarios, sin embargo, esto no evita disputas por el acceso al suelo que puedan derivar en procedimientos inconclusos, desacuerdos entre las partes y fraudes que a su vez generan la irregularidad en la tenencia de la tierra

\section{Conclusiones}

Las modificaciones jurídicas de la tenencia de la tierra en México han abierto nuevas modalidades de acceso al suelo. La formalidad que se pretendía alcanzar con los nuevos mecanismos de privatización no ha funcionado directamente, sino que ha sido transformada y construida por los actores a través de la complementación de acciones formales e informales. Para el estudio de este proceso, la estructura metodológica ofrecida por el marketing ofrece elementos que corresponden con las acciones de compra-venta de suelo ejidal en la periferia urbana, tal como las etapas que atraviesa el proceso (encuentro, negociación, conclusión y vigilancia), 
así como las características del bien, los mecanismos de coordinación, las condiciones, los contratos, los sistemas de vigilancia.

Por su parte, el capital social ofrece elementos para integrar las dimensiones sociales y económicas de las relaciones. En este caso, la definición propuesta permitió evidenciar que durante cada una de las etapas del proceso de compra-venta de tierras parceladas en la periferia, los actores están constantemente construyendo relaciones y acumulando crédito social por medio del intercambio, principalmente, de información. También que los mecanismos de coordinación no sólo se basan en el precio, sino en una serie de promesas y obligaciones adquiridas durante las negociaciones y que el establecimiento de las condiciones de la transacción es moldeado o sustentado por mecanismos como la obligación, la lealtad, la reputación, los objetivos comunes y la confianza. Esto sucede tanto al interior del ejido como al exterior, cuando se tiene contacto con otros grupos, actores y dependencias gubernamentales.

Cabe mencionar que, en teoría, el concepto de capital social serviría para analizar cualquier tipo de relación en la estructura social (ya sea horizontal, vertical o transversal, mercantil o no mercantil). Sin embargo, ello no significa que el capital social deba ser visto como la panacea, por el contrario, las definiciones son múltiples, cuenta con ambigüedades teóricas, tiene limitaciones metodológicas de escala y carece de indicadores proxy que deben ser consideradas durante el análisis. No obstante, al igual que autores como Lomintz, 1988, Coleman, 1995; Portes, 1998; Burt, 1997; Putnam, 1995; Grootaert y Van Bastelaer, 2001 y Ostrom, 2000, en este análisis se verifica que durante el proceso de acceso al suelo en la periferia urbana mediante compra-venta, el capital social mejora el acceso a la información y se reducen las imperfecciones de la misma, otorgando beneficios a los miembros del grupo; concede posiciones de poder más benéficas para la negociación, influye en la capacidad de cálculo del precio, subsana los huecos estructurales, mejora los vínculos existentes en la red propiciando su expansión y permite la modificación de normas y la adaptación a nuevas situaciones, en este caso las mercantiles. Tal es el caso del tránsito entre procedimientos formales e informales que resultan en acciones eficientes para el proceso.

Por el contrario, cabe aclarar que, aun cuando los propósitos de los actores son de interés mutuo, no son necesariamente para el beneficio común (sino personal), lo que puede generar costos que exceden el valor de los beneficios adquiridos o que éstos puedan ser sub-óptimos o que causen importantes externalidades negativas en el futuro mediato o inmediato. Esto es particularmente relevante para los ejidatarios, pues son ellos quienes más dificultad tienen para extender y sostener sus vínculos sociales más allá de aquéllos que generan capital social de unión. 
En cuanto a la etapa de conclusión y vigilancia, las autoridades estatales intervienen como elementos de legitimación de la propiedad y de los contratos formales, al menos como un símbolo. A pesar de que un contrato se haga por medios informales, actores se remiten a marcos jurídicos estatales y formales. Los ejidatarios confían y tienen la expectativa que el órgano estatal vigilará y resolverá los conflictos que puedan derivarse por las transacciones, aunque esto no suceda o tome ańos en solucionarse. Si bien dichos mecanismos alcanzan su propósito de compra-venta de suelo de propiedad social en las periferias urbanas, en el sentido de la planeación y la ocupación del mismo, los resultados no son los más deseables.

\section{Fuentes consultadas}

Abramo, Pedro (2012), "La ciudad confusa: mercado y producción de la estructura urbana en las grandes metrópolis latinoamericanas", Eure, 38 (114), Pontificia Universidad Católica de Chile, Santiago de Chile, Chile, pp. 35-69.

Aoki, Masahiko (2007), "Individual social capital, social networks and the linkages to economic game", The international Bank of Reconstruction and Development, Washington, <http://siteresources. worldbank.org/EXTABCDE/Resources/7455676-1288 210792683/Masahiko-Aoki.pdf>, 11 de enero de 2013.

Appendini, Kirsten y Marcelo de Luca (2006), Género y Trabajo. Estrategias rurales en el nuevo contexto agrícola mexicano, Food and Agriculture Organization, Roma, Italia

Arriagada, Irma (2003), "Capital social: potencialidades y limitaciones analíticas de un concepto", Estudios Sociológicos, XXI (63), El Colegio de México, Ciudad de México, México, pp. 557-584.

Azuela, Antonio (1989), La ciudad, la propiedad privada y el derecho, El Colegio de México, Ciudad de México, México.

Berry, Sara (1989), "Social Institutions and access to resources", Africa: Journal of the International African Institute, 59 (1), Cambridge University Press, Nueva York, Estados Unidos de América, pp. 41-55. 
Bourdieu, Pierre (1986), "The forms of capital”, en John Richardson (ed.), Handbook of Theory Research for the sociology of education, Nueva York, Estados Unidos de América, pp. 47-51.

Bourdieu, Pierre (1980), "Le capital social", Actes de la recherche en sciences sociales, vol. 31, Editions du Seuil, Paris, Francia, pp. 2-3.

Bouquet, Etienne (1999), "Mercado de tierras ejidales en Tlaxcala: formalidad e informalidad del cambio institucional", Revista Estudios Agrarios, 5 (11), Procuraduría Agraria, Ciudad de México, México, pp. 67-106.

Burt, Ronald (1997), "The contingent value of social capital", Administrative Science Quarterly, 42 (2), Sage-Samuel Curtis Johnson Graduate School of Management at Cornell University, Ithaca, Estados Unidos de América, pp. 339-365.

Canabal, Beatriz (2005), "Actores rural-urbanos: proyectos e identidades", en Héctor Ávila (coord.), Lo urbano-rural, ¿nuevas expresiones territoriales?, Universidad Nacional Autónoma de México, Cuernavaca, México, pp. 161-177.

Callon, Michel (2006), "What does it mean to say that economics is performative?", documento de trabajo 005, Centre de Sociologie de l'innovaation, École des Mines de Paris, París, Francia, pp. $1-58$

Coase, Ronald Harry (1960), "The problem of social cost", Journal of Law and Economics, núm. 3, The University of Chicago Press, Chicago, Estados Unidos de América, pp. 1-44.

Coleman, James (1988), "Social capital in the creation of human capital", American Journal of Sociology, vol. 94, The University of Chicago Press, Chicago, Estados Unidos de América, pp. 95-120.

Clichevsky, Nora (2000), Informalidad y segregación urbana en Latino América. Una aproximación, Comisión Económica para América Latina, Santiago de Chile, Chile.

Cruz, María Soledad (2005), "Las dimensiones rural y urbana en los espacios periféricos metropolitanos. El Caso de la Zona Metropolitana del Valle de México", en Héctor Ávila, (coord.), Lo urbano- 
rural ¿nuevas expresiones territoriales?, Universidad Nacional Autónoma de México, Ciudad de México, México, pp.179-206.

Cruz, María Soledad y Martha Chávez (2012), "Procede: privatización y urbanización de la tierra de propiedad ejidal. El caso de la ciudad de Colima”, en María Soledad Cruz (coord.), Periferias metropolitanas, politicas públicas y medio ambiente, Universidad Autónoma Metropolitana, unidad Azcapotzalco-Red de Investigación Urbana, Ciudad de México, México, pp. 103-130.

Duhau, Emilio y Martha Schteingart (2002), "La urbanización popular en la ciudad de México", en Martha Schteingart (coord.), Pobre$z a$, condiciones de vida y salud en la ciudad de México, El Colegio de México, Ciudad de México, México, pp. 29-42.

Duhau, Emilio y Angela Giglia (2008), Las reglas del desorden: habitar la metrópoli. Arquitectura y urbanismo, Siglo XXI, Ciudad de México, México.

Durston, John (2002), El capital social campesino en la gestión del desarrollo rural, Comisión Económica para América Latina, Santiago de Chile, Chile.

Eibenschutz Roberto y Pablo Benlliure (2009), Mercado formal e informal de suelo. Análisis de ocho ciudades, Cámara de Diputados-Secretaría de Desarrollo Social-UAM, unidad Xoxhimilco-Editorial Porrúa, Ciudad de México, México.

Fligstein, Neil y Luke Dauter (2006), "The sociology of markets”, documento de trabajo, Institute for research on Labor and Employment, Departamento de sociología, Universidad de California, Estados Unidos de América, <https://escholarship.org/uc/ item/8qw8cljx>, 21 de abril de 2014.

García-Valdecasas Medina, José (2011), "Una definición estructural del capital social”, Redes: revista hispana para el análisis de redes sociales, 20 (6), Grupo egolab-Grup de Recerca en Antropologia Fonamental i Orientada-LRPC, Sevilla, España, pp.132-160.

Garibay-Orozco, Claudio y Alejandra Balzaretti-Camacho (2009), "Goldcorp y la reciprocidad negativa en el paisaje minero de Mezcala, 
Guerrero", Desacatos, núm. 30, Ciesas, Ciudad de México, México, pp. 91-110.

González, Maya y Lucy Nelly (2004), "El Procede y el Piso en la incorporación del suelo de propiedad social a usos urbanos en los municipios conurbados de la ZMCM", Estudios Demográficos y Urbanos, 19 (56), El Colegio de México, Ciudad de México, México, pp. 313-375.

Grandlgruber, Bruno y Arturo Lara (2007), "Introducción: la teoría económica institucional y evolutiva", en Geoffrey Martin Hodgson, Economía institucional y evolutiva contemporánea, Universidad Autónoma Metropolitana, Ciudad de México, México, pp. 7-23.

Granovetter, Mark (2005), “The impact of social structure on economic outcomes", Journal of Economic Perspectives, 19 (1), American Economic Association, Tennessee, Estados Unidos de América, pp. 33-50.

Granovetter, Mark (1985), "Economic action and social structure: the problem of embeddedness", American Journal of Sociology, 91 (3), The University of Chicago Press, Chicago, Estados Unidos de América, pp. 481-510.

Grootaert, Chistiaan y Thierry Van Bastelaer (2001), "Understanding and measuring social capital: a synthesis of findings and recommendations from the social capital initiative", documento de trabajo núm. 24, Social Capital Initiative, Washington, Estados Unidos de América.

Helmke, Gretchen y Steven Levitsky (2004), "Informal institutions and comparative politics: a research agenda", Perspectives on Politics, 2 (4), Cambridge University Press, Indiana, Estados Unidos de América, pp. 725-740.

Hiernaux, Daniel y Alicia Lindón (1998), "Proceso de ocupación del suelo, mercado de tierra y agentes sociales. El valle de Chalco, Ciudad de México: 1978-1991", en Edith Jiménez Huerta, Análisis del Suelo Urbano (comp.), Instituto Cultural de Aguascalientes, Aguascalientes, México, pp. 225-253. 
Hodgson, Geoffrey (1988), Economics and Institutions, Polity Press, Filadelfia, Estados Unidos de América.

Janvry, Alain y Elisabeth Sadoulet (2001), "Access to land and land policy reforms", documento de trabajo núm. 3, World Institute for Development Economics Research, Universidad de Naciones Unidas, Helsinki, Finlandia.

Jaramillo, Samuel (2008), "Reflexiones sobre la 'informalidad' fundiaria como peculiaridad de los mercados de suelo en las ciudades de América Latina”, Territorios, núm. 18-19, Universidad del Rosario, Bogotá, Colombia, pp.11-53.

Kotler, Philip y Gary Armstrong (2003), Fundamentos del Marketing, Prentice Hall, Ciudad de México, México.

Krishna, Anirudh (2007), "How does social capital grow? A seven-year study of villages in India", Journal of Politics, 69 (4), University of Chicago Press, Chicago, Estados Unidos de América, pp. 941-956.

Le Roy, Étienne (2007), "Le mystère du droit foncier. Sens et non-sens d'une politique volontariste de généralisation de la propriété privée de la terre dans le décollage des économies des sociétés du Sud", en Christoph Eberhard, Enjeux foncier et environnementaux. Dialogues afro-indiens, Pondichery, Francia, pp. 57-88.

Le Roy, Étienne (1991), "Introduction générale”, en Émile le Bris, Etienne Le Roy y Paul Mathieu (eds.), L'appropriation de la terre en Afrique noire. Manuel d'analyse de décision et de gestion foncières, Karthala, París, Francia, pp. 11-22.

Lomnitz-Adler, Larissa y Diana Sheinbaum (2011), "De redes sociales recíprocas a grupos de acción para el intercambio de mercado: la 'privatización espontánea' en la Hungría post-comunista', Redes, Revista Hispana para el análisis de redes sociales, vol. 21, Grupo egolab-Grup de Recerca en Antropologia Fonamental i Orientada-LRPC, Sevilla, España, pp. 538-570.

Lomnitz-Adler, Larissa (1988), "Informal exchange networks in formal systems: a theorical model”, American Anthropologist, 90 (1), American Anthropological Association, Arlington, Estados Unidos de América, pp. 42-55. 
Lunn, Peter D. (2012), "Behavioral economics and policymaking: learning from the early adopters", The economic and Social Review, 43 (3), The Economic and Social Research Institute, Dublin, Irlanda, pp. 423-449.

Mauss, Marcel (1971), Ensayo sobre los dones: razón y forma del cambio en las sociedades primitivas. Sociología y Antropología, Editorial Tecnos, Madrid, España.

Morales-Schechinger, Carlos (2012), "Curar o vacunar, dos políticas en tensión: la regularización frente a las reservas territoriales en el desarrollo urbano sustentable", en Clara Eugenia Salazar-Cruz (coord.), Irregular: suelo y mercado en América Latina, El Colegio de México, Ciudad de México, México, pp. 125-158.

Narotsky, Susana (2004), Antropología Económica. Nuevas tendencias, Editorial Melusina, Barcelona, España.

Narayan, Deepa (1999), "Bonds and bridges: social capital and poverty. Poverty group”, working paper WPS2167, The World Bank, Washington, Estados Unidos de América.

North, Douglas (1995), Instituciones, cambio institucional y desempeño económico, Fondo de Cultura Económica, Ciudad de México, México.

Ostrom, Elinor (2000), "collective action and the evolution of social norms", The Journal of Economic Perspectives, 14 (3), American Economic Association, Tennessee, Estados Unidos de América, pp. 137-158.

Payne, Geoffrey (2004), "Land tenure and property rights", Habitat International, 28 (2), Elsevier, Nueva York, Estados Unidos de América, pp. 167-316.

Portes, Alejandro (2013), Sociología económica. Una investigación sistemática, Centro de Investigaciones Sociológicas, Madrid, España.

Portes, Alejandro (2006), "Instituciones y desarrollo: una revisión conceptual”, Cuadernos de Economía, 25 (45), Facultad de Ciencias Económicas de la Universidad Nacional de Colombia, Bogotá, Colombia, pp. 13-52. 
Portes, Alejandro y Bryan Roberts (2005), "La ciudad bajo el libre mercado", en Bryan Roberts, Alejandro Portes y Alejandro Grimson (eds.), Ciudades Latinoamericanas: un análisis comparativo en el umbral del nuevo siglo, Prometeo, Buenos Aires, Argentina, pp. 19-74.

Portes, Alejandro (1998), "Social capital: its origins and application in modern sociology”, Annual Review of Sociology, vol. 24, Annual Reviews, Palo Alto, Estados Unidos de América, pp. 1-24.

Putnam, Robert (1995), "Bowling alone: America's declining social capital", Journal of Democracy, VI (1), Johns Hopkins University Press, Washington, Estados Unidos de América, pp. 65-78

Rème, Pétronille (2005), "El Mercado de los economistas y el Mercado de los sociólogos", Cuadernos de Economía, XXIV (43), Elsevier, Bogotá, Colombia, pp. 13-34.

Requena-Santos, Félix (1989), "El concepto de red social”, REIS. Revista Española de Investigaciones sociológicas, 48 (89), Centro de Investigaciones Sociológicas, Madrid, España, pp. 137.

Robles-Berlanga Héctor Manuel (2008), "Saldos de las reformas de 1992 al artículo 27 constitucional”, Estudios Agrarios, Revista de la Procuraduría Agraria, núm. 38, Procuraduría Agraria, Ciudad de México, México, pp. 131-150.

Rutherford, Malcom (2003), "La economía institucional antes y ahora", Revista Análisis Económico, XVIII (38), Universidad Autónoma Metropolitana, Unidad Azcapotzalco, Ciudad de México, México, pp.13-39.

Salazar-Cruz, Clara Eugenia (2014), "El puño invisible de la privatización”, Territorios, núm. 30, Universidad del Rosario, Bogotá, Colombia, pp. 69-90.

Salazar-Cruz, Clara Eugenia (2012), "Los ejidatarios en el control de la regularización”, en Clara Eugenia Salazar-Cruz (coord.), Irregular: suelo y mercado en América Latina, El Colegio de México, Ciudad de México, México, pp. 265-306.

Salazar-Cruz, Clara Eugenia (2009), "La disponibilidad de suelo social en las áreas metropolitanas del país", Estudios Agrarios, Revista de 
la Procuraduría Agraria, 15 (41), Procuraduría Agraria, Ciudad de México, México, pp.125-148.

Schteingart, Martha (1989), Los productores del espacio habitable. Estado, empresas y sociedad en la Ciudad de México, El Colegio de México, Ciudad de México, México.

Steiner, Philippe (2009), "Échanges, transactions et lien social", documento de trabajo núm. 11, Socious, Centro de Investigación en Sociología Económica y de las Organizaciones, Lisboa, Portugal.

Williamson, Oliver E. (1981) "The economics of organization: the transaction cost approach", American Journal of Sociology, 87 (3), The University of Chicago Press, Chicago, Estados Unidos de América, pp. 548-577.

Woolcock, Michael (2012), "Prefacio", en Patricia López-Rodríguez e Isidro Soloaga (eds.), Capital Social y política pública en México, El Colegio de México-Instituto Nacional de las Mujeres, Ciudad de México, México, pp. 9-11.

Woolcock, Michael (2001), "The place of social capital in understanding Social and Economic Outcomes", Canadian Journal of Policy Research, 1 (2), Les presses de l'Université de Montréal, Montreal, Canadá, pp. 11-17.

Woolcock, Michael (1998) "Social capital and economic development, toward a theoretical synthesis and policy framework", Theory and society, 2 (27), Springer, Los Angeles, Estados Unidos de América, pp. 151-208.

Yúnez-Naude, Antonio (2006) "Liberalización y reformas al agro: lecciones de México", Economía Agraria y Recursos Naturales 6 (12), Centro de Estudios Económicos-El Colegio de México, Ciudad de México, México, pp. 47-67.

Recibido: 7 de octubre de 2015.

Corregido: 19 de octubre de 2016. Aceptado: 22 de enero de 2017. 
Sandra Pola-Villaseñor. Doctoranda en Geografía por la Universidad Nacional Autónoma de México. Maestra en Estudios Urbanos por el Colegio de México, A. C. Realizó un intercambio académico en la Universidad de Montreal en Faculté de l'Aménagement. Sus principales líneas de investigación son: Urbanización en la periferia, acceso, mercado, gestión del suelo y tierras ejidales. Entre sus publicaciones se encuentran: Expansión urbana y formas de la incorporación de tierras de origen social al desarrollo urbano antes y después de las reformas al articulo 27 constitucional caso de Morelia, Michoacán, tesis de maestría, El Colegio de México, Ciudad de México, México (2009).

Yadira Méndez-Lemus. Doctora en Planeación para el Desarrollo por la Universidad de Queensland, Australia. Actualmente es profesora-investigadora en el Centro de Investigaciones en Geografía Ambiental, Universidad Nacional Autónoma de México, campus Morelia, así como secretaria académica del mismo. Sus principales líneas de investigación son: Pobreza, vulnerabilidad y adaptación y Procesos territoriales y dinámicas sociales en las periferias urbanas. Entre sus últimas publicaciones destacan, en coautoría: "Tracing processes in poverty dynamics: a tale of peri-urban small-scale farmers in Mexico City", Urban Studies, 51 (10), Sage, pp. 2009-2035 (2014); en coautoría, "Procesos participativos intramunicipales como pasos hacia la gobernanza local en territorios periurbanos. La experiencia en el municipio de Tarímbaro, Michoacán, México", Journal of Latin American Geography, 14 (2), Univesrsity of Texas Press, Project Muse, pp. 129-157 (2015); en coautoría, "Peri-urban local governance? Intra-government relationships and social capital in a peripheral municipality of Michoacán, Mexico", Progress in development Studies, 17 (1), Sage, pp. 1-23 (2017).

Antonio Vieyra. Doctor en Geografía Humana con la distinción Cum Laude en la Universidad Complutense de Madrid, donde además obtuvo la especialidad en Trabajo, empleo y desarrollo. Actualmente es profesorinvestigador titular definitivo en el Centro de Investigaciones en Geografía Ambiental, Universidad Nacional Autónoma de México, campus Morelia; ha sido profesor de diversos cursos de posgrado y licenciatura en la Universidad Nacional Autónoma de México. Es miembro del Sistema nacional de Investigadores, nivel I. Ha participado en 21 proyectos de investigación y sus resultados han sido publicados en más de 48 publicaciones primarias, nacionales e internacionales, entre artículos, libros y capítulos de libro. Sus principales líneas de investigación son: Análisis urbano-regional, urbanización y ambiente. Entre sus últimas publicaciones destacan, en coautoría: "Spatial fix and metabolic rift as conceptual 
tools in land-change science", Capitalism Nature Socialism, 26 (4), Taylor and Francis Online, pp. 198-214 (2015); co-coordinador de Procesos urbanos, pobreza y ambiente. Implicaciones en ciudades medias y megaciudades, UNAM, Ciudad de México, México (2016); "Informality, migration, and geographic rift in Latin America”, en Hernández, Felipe y Axel Becerra (co-ed.), Viewing Latin American Cities, Cambridge Scholars Publishing, pp. 48-63 (2017). 